\title{
Short Term Evaluation of Warfarin Versus Rivaroxaban Regimens in the Treatment of Deep Venous Thrombosis
}

\author{
R.F.Shnouda ${ }^{1}$, A.E.Mohamed ${ }^{2}$, Sh.A.Mostafa ${ }^{2}$, A.A.Katta ${ }^{1}$ and H.M.Aboul-Enien ${ }^{2}$ \\ ${ }^{1}$ National Heart Institute, Imbaba, Egypt \\ ${ }^{2}$ CardiologyDept., Faculty of Medicine, Benha Univ., Benha, Egypt \\ E-Mail:Reham@gmail.com
}

\begin{abstract}
The Low molecular weight heparin (LMWH) and vitamin K antagonists (VKAs) were considered as the first line management option for the prevention and treatment of deep venous thromboembolism for several decades as warfarin was the only oral anticoagulation option; but new oral anticoagulants have the potential to change the management of coagulation disorders. As they differ from VKAs in their anticoagulation mechanism because of direct inhibition of proteins of the coagulation cascade. They have more predictable pharmacokinetics that leads to a fixed and convenient dosing regimens with no need for routine monitoring, as well as in a rapid onset of action, and high efficacy and low risk of bleeding. Their limitations are their higher cost, limited monitoring (if needed, as only qualitative measures available) and the lack of a specific antidote. This study aims to study the short term outcome of two regimens Rivaroxaban versus warfarin in acute deep venous thrombosis treatment regarding their rate of recanalization using venous duplex. This work was done over 100 patients, divided into 2 equal groups: Group received Rivaroxaban for 1 month, and group 2 received warfarin. The following was done for all patients: creatinine venous duplex at time of diagnosis and another follow up after one month and rate of recanalization was assessed. Rivaroxaban (group 1) included 25 males and 25 females with a mean age of 54.14 (29-81), and a mean BMI of $24.64 \pm 3.73$. Warfarin (group 2) included 24 males and 26 females with a mean age of 52.04 (28- 80), and a mean BMI of 23.64 \pm 3.57 . There were $22 \%$ in the Rivaroxaban group had no or minimal recanalization versus $70 \%$ in the warfarin group. While $78 \%$ had partial to complete recanalization in the Rivaroxaban group versus $30 \%$ in the warfarin group with a statistical significant differences between the 2 arms of the study. Rivaroxaban showed a better rate of recanalization compared to warfarin in short term follow up of patients with acute DVT.
\end{abstract}

Keywords: Rivaroxaban, Warfarin, Venous thromboembolism, Recanalization.

\section{Introduction}

Novel Oral Anticoagulants (NOAC) are used as an alternative to Vitamin K Antagonist (VKA). The European Society of Cardiology guidelines recommend the use of NOACs as alternatives to VKA anticoagulation for secondary prevention of Venous Thromboembolism (VTE). NOAC help in overcoming the complications of overlapping a parenteral anticoagulant with a VKA. NOACs have been shown to be effective in the prevention and treatment of VTE [1].

The NOACs offer advantages of predictable pharmacokinetic profile and lesser food and drug interactions compared to warfarin. Moreover NOACs do not require routine monitoring. The NOACs have been extensively studied in the treatment of acute VTE and prevention of VTE. Dabigatran, Rivaroxaban, apixaban and edoxaban have shown to be effective and at least comparable in safety with the conventional treatment of acute VTE. They are associated with lower risk of intracranial or intracerebral bleeding compared to warfarin [4].

The purpose of anticoagulation is the prevention of recurrent thrombosis, embolization, and death, the risk of which is greatest in the first three to six months following the diagnosis [3].

This study aims to study the short term outcome of two regimens Rivaroxaban versus warfarin in acute deep venous thrombosis treatment regarding their rate of recanalization using venous duplex.

\section{Methodology}

All patients were subjected to the following:

Full Medical History: with special emphasis on risk factors such as: Age \& gender, smoking, family history, Hypertension, Diabetes Mellitus, history of acquired causes of DVT Current medical treatment and history of drug allergy and Complaint: Lower limb swelling, major or minor bleeding.

Also to a full clinical examination: with special emphasis on BMI, Vital signs, Bruises and ecchymosis And Lower limb swelling.

Laboratory investigations: Include the following serum creatinine level, INR test and time to target INR was calculated to the warfarin group.

All patients had a venous duplex at the time of diagnosis: showed acute or sub-acute DVT with total occlusion and level of obstruction was determined.

Patients were divided into two groups after been diagnosed with Acute or sub-acute DVT into:

\section{First group}

Iincludes 50 patients treated with $15 \mathrm{mg}$ of Rivaroxaban every 12 hours for 21 days and then $20 \mathrm{mg}$ of Rivaroxaban once daily.

\section{Second group}

Includes 50 patients treated with Warfarin with Enoxaparin with dose of $1 \mathrm{mg} / \mathrm{kg}$ subcutaneous every 12 hours till target INR (2-3) was achieved then Warfarin was given alone. 
A Follow up venous duplex was done to all patients one month from the time of diagnosis and assessment of degree of recanalization.

Rivaroxaban (group 1) included 25 males and 25 females with a mean age of 54.14 (29-81), and a mean
BMI of $24.64 \pm 3.73$. Warfarin (group 2) included 24 males and 26 females with a mean age of 52.04 (28$80)$, and a mean BMI of $23.64 \pm 3.57$.

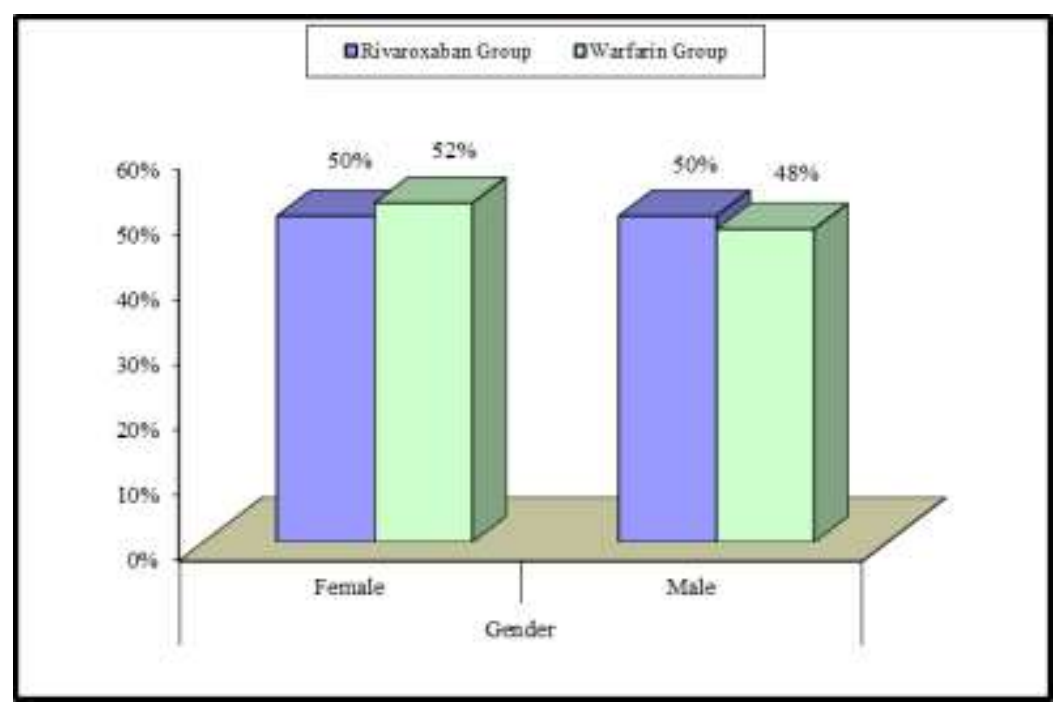

Fig (1) Comparison between rivaroxaban group and warfarin group regarding gender of the studied patients.

There were $22 \%$ of patients in the Rivaroxaban group had no or minimal recanalization versus $70 \%$ of patients in the warfarin group. While $78 \%$ of patients had partial to complete recanalization in the
Rivaroxaban group versus $30 \%$ of patients in the warfarin group with a statistical significant differences between the 2 arms of the study p value $<0.001$.

Table (1) AComparison between Rivaroxaban group and Warfarin group regarding rate of recanalization.

\begin{tabular}{|c|c|c|c|c|c|c|c|}
\hline & \multicolumn{2}{|c|}{$\begin{array}{c}\text { Rivaroxaban } \\
\text { Group }\end{array}$} & \multicolumn{2}{|c|}{$\begin{array}{c}\text { Warfarin } \\
\text { Group }\end{array}$} & \multirow[t]{2}{*}{ Test value } & \multirow[t]{2}{*}{ P-value } & \multirow[t]{2}{*}{ Sig. } \\
\hline & No. & $\%$ & No. & $\%$ & & & \\
\hline No to minimal recanalization & 11 & $22.0 \%$ & 35 & $70.0 \%$ & \multirow{2}{*}{23.188} & \multirow{2}{*}{$<0.001$} & \multirow{2}{*}{ HS } \\
\hline Partial to complete recanalization & 39 & $78.0 \%$ & 15 & $30.0 \%$ & & & \\
\hline
\end{tabular}

P-value >0.05: Non significant (NS); P-value <0.05: Significant (S); P-value< 0.01: highly significant (HS) *:Chi-square test.

\section{Discussion}

That was concordant with [6] showed patients who received oral Rivaroxaban displayed a lower incidence of Posthrombotic syndrome and a better total vein recanalization rate after 6 and 12 months than patients who received warfarin.

And also concordant with [2], showed that Rivaroxaban have the advantage of more vascular outcomes compared with warfarin because recanalization occurred, with high percentage in new oral anticoagulants (Rivaroxaban) more than warfarin.

And disconcordant with [5], Who stated that Treatment of DVT with Rivaroxaban or warfarin was effective and there was no difference in the number of segments that remained involved after 180 days' follow-up of both groups. However, patients treated with Rivaroxaban exhibited evidence of earlier recanalization than patients treated with warfarin. Whoever it this study had a prolonged follow up 6 month and as it showed earlier recanalization it may be as same as our study which showed better recanalization rate after one month.

\section{Conclusion}

Rivaroxaban showed a better rate of recanalization compared to warfarin in short term follow up of patients with acute DVT.

\section{References}

[1] T.Mohammed, Y. Mohamed, M.Khaled. Evaluation of new oral anticoagulation in management of deep vein thrombosis. Al Azhar Med. J., , Vol.46(2), PP.433-442,2017.

[2] S.Rafael, Marcelo, C.Francisco. Comparison of the recanalization rate and postthrombotic syndrome in patients with deep venous thrombosis treated with rivaroxaban or warfarin, Surgurey Vascular, Vol.166, pp.1076-1083,2019. 
[3] P. Polyana, P. Aline, Danielle Oliveira de Andrade. Analysis of recanalization of deep venous thrombosis: a comparative study of patients treated with warfarin vs. Rivaroxaban, Vasc Bras, Vol.17, PP.1677-5449,2017.

[4] M.Martin, M.Khanimana , What's the Best Anticoagulation Management for Deep Venous Thrombosis?. Clin Sur, Vol.4, PP.2334,2019.
[5] A. Pipilis, Management of Bleeding with Newer Anticoagulants. Hosp Chron, Vol.9(1), PP.546,2014.

[6] T.L. Ortel, I .Neumann, W. Ageno, American Society of Hematology 2020 guidelines for management of venous thromboembolism: treatment of deep vein thrombosis and pulmonary embolism. Blood Adv, Vol.4(19), PP.4693738,2020 . 